\title{
Kasachstans Konnektivitätsambitionen in der OSZE: Handelsförderung und Normumgehung
}

\author{
Sebastian Mayer
}

\section{Zusammenfassung}

In den 2010er Jahren wurde „Konnektivität“ als politisches Instrument zu verbesserten Wirtschaftsbeziehungen zum Schlagwort in der zweiten Dimension (Wirtschafts- und Umweltdimension) der OSZE. Kasachstan hat vorgeschlagen, in der Hauptstadt des Landes ein OSZEKonnektivitätszentrum zu errichten, welches entsprechendes Fachwissen bereit stellen soll. Dieser Beitrag erörtert das Konzept der Konnektivität, beschreibt die Konnektivitätsagenda Kasachstans und beleuchtet die dahinterstehenden Motive. Diese Initiative ist nicht nur durch die Binnenlage des Landes motiviert, sondern auch durch den Wunsch, Verpflichtungen aus der ersten (politisch-militärischen) und insbesondere aus der dritten (menschlichen) Dimension der OSZE abzuschwächen. Mit Verweis auf die potenzielle Rolle der OSZE im Bereich der Konnektivität, aber auch auf Kasachstans bedeutende Beiträge zur regionalen und internationalen Kooperation und Stabilität, zeigt dieser Beitrag, dass die kasachische Initiative ernstgenommen werden sollte. Allerdings darf nicht zugelassen werden, dass die drei Sicherheitsdimensionen der OSZE gegeneinander ausgespielt werden. Kasachstan muss seinen Verpflichtungen aus der ersten und dritten Dimension auch weiterhin nachkommen.

\section{Schlagworte}

Konnektivität, Wirtschaftsbeziehungen, OSZE, Zentralasien, Kasachstan, Handelsförderung

Bitte zitieren als: Sebastian Mayer, Kasachstans Konnektivitätsambitionen in der OSZE: Handelsförderung und Normumgehung, OSCE Insights 4 (Baden-Baden: Nomos, 2021), https://doi.org/10.5771/9783748911630-04

\section{Einführung}

In den 2010er Jahren erlangte der Begriff „Konnektivität“ große Beliebtheit. Er bezeichnet ein politisches Instrument, das insbesondere die Wirtschaftsbeziehungen zwischen den Transitionsländern

* Dr. Sebastian Mayer

Deutsch-Kasachische Universität

Almaty, Kasachstan

mayer@dku.kz
Südost- und Osteuropas sowie der ehemaligen Sowjetunion verbessern soll. Obwohl die Verbesserung der Wirtschaftsbeziehungen bereits im zweiten Kapitel der Schlussakte von Helsinki aus dem Jahr 1975 erwähnt wird, verwendete die OSZE den Begriff Konnektivität erst während des deutschen OSZE-Vorsitzes im Jahr 2016. Die OSZE arbeitet laut eigener Darstellung daran, über einen Dialog zu Handel und Verkehr eine Grundlage für wirtschaftliche Konnektivität zwi- 
schen den 57 Teilnehmerstaaten zu schaffen. ${ }^{1}$

Vor allem Kasachstan hat das Thema Konnektivität in den Mittelpunkt seiner Außenpolitik gestellt, was angesichts der Binnenlage und der fehlenden Anbindung Kasachstans an die globalen Märkte nicht überrascht. Die kasachische Regierung hat für die Idee geworben, in der Hauptstadt Nur-Sultan ein von der OSZE geführtes Themenzentrum für Konnektivität einzurichten. Mit einem solchem Kompetenzzentrum könnte das Land von entsprechendem Fachwissen profitieren. Kasachstan hat jedoch noch weitere Gründe, sich für Konnektivität als Schwerpunktthema der OSZE einzusetzen. Ein solcher Schwerpunkt würde nämlich die internationalen und regionalen Führungsambitionen Kasachstans befördern. Auch böte eine vorrangige Behandlung der zweiten OSZE-Dimension (Wirtschaft und Umwelt) eine willkommene Gelegenheit, ungewollte Verpflichtungen aus den beiden anderen OSZEDimensionen, der politisch-militärischen und der menschlichen Dimension, herunterzuspielen. Vor allem im Hinblick auf die menschliche Dimension erweisen sich kasachische Staatsvertreter nach wie vor als reformresistent, insbesondere, wenn es um Grundrechte, Wahlen und die Unabhängigkeit der politischen Parteien geht. Auch besteht Grund zu der Annahme, dass die kasachische Regierung zumindest mittelfristig beabsichtigt, das derzeitige OSZE-Programmbüro in Nur-Sultan, das sich allen drei Dimensionen widmet, durch das vorgeschlagene Konnektivitätszentrum, das sich nur mit der zweiten Dimension befassen würde, zu ersetzen.

Dieser Beitrag untersucht das Konzept der Konnektivität im Allgemeinen sowie im OSZE-Kontext, stellt die Konnektivitätsagenda Kasachstans dar, beschreibt die dahinterstehenden Motive und formuliert Empfehlungen für die OSZE. Diese sollte die kasachische Initiative ernst nehmen, weil die OSZE tatsächlich eine größere Rolle bei der Konnektivität spielen kann. Auch sollten Kasachstans Beiträge zur regionalen und internationalen Kooperation und Stabilität anerkannt werden. Gleichwohl dürfen die drei Dimensionen durch Konnektivität nicht gegeneinander ausgespielt werden. Kasachstan sollte seine Verpflichtungen aus der ersten und dritten Dimension auch weiterhin ernst nehmen.

Der Beitrag stellt zunächst das Konzept der Konnektivität vor. Der zweite Abschnitt präzisiert, wie Konnektivität in der OSZE verankert ist. Darauf folgen zwei Abschnitte, die veranschaulichen, wie Kasachstan den Schwerpunkt auf die Konnektivität legt und welche Motive dahinterstehen. Ausgehend von dieser Analyse unterbreitet der letzte Abschnitt Empfehlungen für die OSZE. ${ }^{2}$

\section{Das Konzept der Konnektivität}

„Konnektivität“, so wie der Begriff in diesem Beitrag verwendet wird, beschreibt die bewusste Gestaltung von Wirtschaftsbeziehungen zwischen Staaten oder Regionen. ${ }^{3}$ Als Instrument wird sie von einzelnen Staaten und internationalen Organisationen in der Wirtschaftsdiplo- 
matie eingesetzt. Während frühe Arbeiten zu zwischenstaatlichen Beziehungen, wie etwa von Keohane und Nye, die verstärkten wirtschaftlichen Interaktionen zwischen den damaligen Industrienationen in den Blick nahmen, ${ }^{4}$ konzentrierten sich Forscher*innen nach der Wende von 1989 vermehrt auf die Volkswirtschaften des ehemaligen sozialistischen Blocks. Für diese Staaten war Konnektivität ein wichtiger Motor des wirtschaftlichen Fortschritts. Beispiele für bisherige Konnektivitätsinitiativen sind u.a. das 1991 von der damaligen Europäischen Gemeinschaft ins Leben gerufene und inzwischen ausgelaufene TACIS-Programm (Technical Assistance to the Commonwealth of Independent States), die 2013 von China angekündigte Belt and Road Initiative (BRI), die japanische Initiative Partnership for Quality Infrastructure aus dem Jahr 2015 und die EU-Asien-Konnektivitätsstrategie der Europäischen Union (EU), die 2018 gestartet wurde. Neben ihren konkreten materiellen Vorteilen kann Konnektivität als vertrauensbildende Maßnahme die friedlichen Beziehungen zwischen den beteiligten Staaten fördern. ${ }^{5}$ Diese Erwartungen entsprechen einem liberalen Denkansatz, nach dem wirtschaftlicher Austausch Stabilität und Frieden begünstigt.

Staaten unterstützen Konnektivitätsprojekte, weil diese ihren materiellen Interessen und/oder umfassenderen Zielen dienlich sind. Neben Vorteilen birgt Konnektivität aber auch Risiken. Wie Keohane und Nye betonen, können Länder durch verstärkte Wirtschaftsbeziehungen in unterschiedlichem Maße gefährdet oder geschwächt werden. ${ }^{6}$ Bei- spiele hierfür sind die Erdölembargos der 1970er Jahre, Handelskriege und die Unterbrechungen der Lieferketten durch die COVID-19-Pandemie der vergangenen Monate. Daneben können unterschiedliche Präferenzen hinsichtlich der Verortung und Gestaltung der Wirtschaftsbeziehungen zu Spannungen und zu einer geopolitischen Aufladung des Themas führen. ${ }^{7}$ Hinzu kommen die sehr unterschiedlichen Konnotationen des Begriffs „Konnektivität“, welche Konfliktpotential bergen. Autokratische politische Systeme - allen voran China und seine BRI - gehen meist von einem nicht-normativen, materiellen Konnektivitätsbegriff aus. Der Schwerpunkt liegt dabei auf der physischen Infrastruktur und dem damit verbundenen strategischen Kalkül. In liberalen Staaten und internationalen Institutionen ist das Konzept hingegen tendenziell mit normativen Ansprüchen verbunden, wozu auch die oben erwähnten liberalen Friedenserwartungen gehören. Auch verwandte Konzepte wie gute Regierungsführung (good governance) und Nachhaltigkeit (sustainability) beinhalten eine Reihe von Leitprinzipien, Werten und Normen, die von den übergreifenden Konnektivitätszielen gedeckt sind. Derartige Prinzipien werden zugleich von der OSZE formuliert, auf die wir im nächsten Abschnitt näher eingehen wollen.

\section{Die OSZE und Konnektivität}

Die Bedeutung der Konnektivität für die OSZE geht auf die Schlussakte von Helsinki aus dem Jahr 1975 zurück, deren 
zweites Kapitel die Zusammenarbeit in den Bereichen Wirtschaft und Umwelt thematisiert. Mehrere wegweisende Dokumente aus der Zeit nach dem Ende des Kalten Krieges, die sich auf die zweite Dimension beziehen, bereiteten danach den Boden für dieses Handlungsfeld. Das Schlussdokument der Konferenz über wirtschaftliche Zusammenarbeit in Europa aus dem Jahr 1990 in Bonn fordert ein "dauerhaftes Wirtschaftswachstum" und die „wirtschaftliche Zusammenarbeit“. Außerdem werden die „Rechtsstaatlichkeit und [der] für alle gleiche Schutz durch das Gesetz auf der Grundlage der Achtung der Menschenrechte sowie eines wirksamen, allgemein zugänglichen und gerechten Rechtswesens“ als flankierende Elemente von Wirtschaftswachstum hervorgehoben. ${ }^{8}$

Bei dem Ministerratstreffen in Maastricht (2003) wurde vor dem Hintergrund erheblicher Schwierigkeiten bei wirtschaftlichen Transformationsprozessen in den ehemals sozialistischen Volkswirtschaften das zentrale OSZE-Strategiedokument für die Wirtschafts- und Umweltdimension (Maastrichter Strategiedokument) verabschiedet. Darin wurden verschiedene Probleme bei der Regierungsführung benannt, etwa ,ineffiziente Institutionen und eine schwache $\mathrm{Zi}$ vilgesellschaft, das Fehlen von Transparenz und Rechenschaftspflicht im öffentlichen und privaten Sektor, [...] Schwächen in der öffentlichen Verwaltung und eine nicht nachhaltige Nutzung natürlicher Ressourcen, Korruption und Missachtung der Unternehmensethik und der Grundsätze einer verantwortungsvollen Unternehmensführung."
Auf den Treffen des Ministerrats im Jahr 2006 (Brüssel) und 2011 (Vilnius) wurden ebenfalls Beschlüsse zur wirtschaftlichen Zusammenarbeit gefasst. Als das Thema im Jahr 2014 unter dem Schweizer Vorsitz ganz oben auf die OSZE-Agenda gesetzt wurde, gewann es erheblich an Dynamik. In dem Beschluss über die Stärkung guter Regierungsführung und die Förderung der Konnektivität, der 2016 auf dem Treffen des Ministerrats in Hamburg gefasst wurde, wird erstmals das Schlagwort „Konnektivität“ im OSZE-Kontext erwähnt. Ferner wird festgestellt, dass "gute Regierungsführung, Transparenz und Rechenschaftspflicht grundlegende Voraussetzungen für Wirtschaftswachstum, Handel, Investitionen und nachhaltige Entwicklung sind und somit zur Stabilität, Sicherheit und Achtung der Menschenrechte [...] beitragen." Die OSZE-Institutionen werden aufgefordert, die Normen und Verfahren für den Handel im OSZE-Raum $\mathrm{zu}$ vereinheitlichen, zu vereinfachen und zu straffen. $\mathrm{Zu}$ den neueren Dokumenten, die das Thema Konnektivität aufgreifen, gehören der Ministerratsbeschluss über die Förderung der wirtschaftlichen Teilhabe im OSZE-Raum von 2017 und die Ministerratserklärung zur digitalen Wirtschaft als treibende Kraft für die Förderung von Zusammenarbeit, Sicherheit und Wachstum aus dem Jahr 2018.

All diese Dokumente basieren auf der Grundannahme, dass die Förderung des wirtschaftlichen Wohlstands und ähnliche Maßnahmen im Kontext der Konnektivität die Sicherheit und Stabilität im OSZE-Raum erhöhen. Jedoch verwendet die OSZE angesichts der verschiedenen 
Schwerpunkte und unterschiedlichen Regierungsformen in den Teilnehmerstaaten bewusst ein vielschichtiges Konnektivitätskonzept. Daher besteht kein Konsens darüber, wie Konnektivität im Rahmen der OSZE genau zu definieren ist.

Innerhalb des institutionellen Rahmens der OSZE fällt Konnektivität in den Zuständigkeitsbereich des Büros des Koordinators für ökonomische und ökologische Aktivitäten der OSZE (OCEEA). Dessen Mandat ergibt sich aus den oben genannten Beschlüssen und Erklärungen, insbesondere dem Maastrichter Strategiedokument von 2003. Die 22 Mitarbeiter des Koordinators decken das gesamte Spektrum der Tätigkeit der OSZE in der zweiten Dimension ab, einschließlich der Konnektivität und anderer eng damit verbundener Bereiche wie Digitalisierung und gute Regierungsführung. ${ }^{10}$

Die OSZE konzentriert sich auf dem Gebiet der Konnektivität geographisch auf Osteuropa, Südosteuropa, den Südkaukasus und Zentralasien. Wie auch in anderen Handlungsbereichen der OSZE erfüllt das OCEEA sein Mandat in Bezug auf die wirtschaftliche Zusammenarbeit der Staaten zum Großteil dadurch, dass es politischen Entscheidungsträgern und Praktikern bewährte Vorgehensweisen aufzeigt. Beispielsweise hat es in Zusammenarbeit mit der Wirtschaftskommission der Vereinten Nationen für Europa (UNECE) ein Handbuch der Best Practices für Grenzkontrollen ${ }^{11}$ und einen Tagungsbericht des Forums für Sicherheit im Binnenverkehr ${ }^{12}$ herausgegeben. Das OCEEA organisiert zudem Workshops, Seminare und Online-Veranstaltungen zu Konnektivitätsthemen auf nationaler und regionaler sowie höchster Verwaltungsebene. Das Themenspektrum der bisherigen Veranstaltungen reichte von grenzüberschreitendem E-Commerce über Transitkoordination bis hin zu papierlosem Handel. So fand im Juni 2020 ein virtuelles Treffen mit dem Titel „Safer and Sustainable Supply Chains Connecting Central Asia and Europe" statt. Ob und in welchem Ausmaß das von Kasachstan vorgeschlagene Konnektivitätszentrum solche OCEEA-Funktionen übernehmen könnte, ist noch unklar.

\section{Kasachstans Konnektivitätsambitionen für die OSZE}

In den Binnenländern Zentralasiens, die zu den am wenigsten verbundenen Gebieten der Welt gehören, kommt Konnektivität und namentlich dem Transportwesen nicht zuletzt wegen der hohen Transitkosten eine besondere Bedeutung zu. ${ }^{13}$ Die kasachische Außenpolitik hat das Thema Konnektivität schon länger priorisiert, und zwar in den letzten Jahren mit steigender Tendenz. Als Mitglied der Eurasischen Wirtschaftsunion (EAWU) ist das Land daran interessiert, den Handel mit anderen EAWU-Mitgliedern zu fördern. Kasachstan unterstützt zudem mit Nachdruck die chinesische BRI, die 2013 in der kasachischen Hauptstadt verkündet wurde. Für die BRI ist Kasachstan ein strategisch wichtiger Korridor im Verkehr zwischen China und dem Westen. Die BRI hat ebenfalls das Potenzial, den Transit unter den EAWUMitgliedern zu verbessern. ${ }^{14}$ Die kasachische Regierung hat ihr eigenes Infrastruk- 
turprogramm Nurly Zhol (Heller Weg), das den Schwerpunkt auf Schienen- und Autobahnverbindungen legt, eng mit der BRI verknüpft. ${ }^{15}$

Vor diesem Hintergrund bevorzugt Kasachstan vor allem Projekte im Bereich der Konnektivität, weil diese zur Modernisierung bestehender Routen und zur Diversifizierung der kasachischen Wirtschaft durch die Entwicklung neuer Handelsrouten beitragen. Vornehmlich lieBen sich dadurch Transportkosten senken und Einnahmen aus Transitgebühren erzielen. Eine bessere Anbindung könnte auch das Ungleichgewicht bei den Exporten beheben, die zurzeit auf wenige Märkte beschränkt sind. Kasachs$\tan$ ist für China als Transportkorridor sowohl für Importe als auch Exporte von entscheidender Bedeutung und darüber hinaus eine Bezugsquelle für Rohstoffe wie Öl und Uran. Jedoch verlief die Kooperation mit der BRI und anderen Konnektivitätsprojekten nicht immer problemlos: Es kam wiederholt zu Problemen aufgrund mangelnder Standardisierung des grenzüberschreitenden Handels und Transits ebenso wie aufgrund eines Defizits an Transparenz, Rechtsstaatlichkeit und Verantwortlichkeit sowie der Anfälligkeit der Hilfsleistungsempfänger für Überschuldung. ${ }^{16} \mathrm{Wie}$ im letzten Abschnitt näher ausgeführt wird, sind dies Problembereiche, mit denen sich ein Konnektivitätszentrum der OSZE befassen könnte.

Während des kasachischen OSZE-Vorsitzes im Jahr 2010 nahm die Regierung des Landes erstmalig die Gelegenheit wahr, die wirtschaftliche Zusammenarbeit ganz oben auf die Tagesordnung der Organisation zu setzen, wobei sie dabei dem Transport auf dem Landweg Priorität einräumte. ${ }^{17}$ Eine weitere Chance ergab sich für die Regierung, als der Schweizer OSZE-Vorsitz - als Reaktion auf frühere Anfragen mehrerer osteuropäischer und zentralasiatischer Teilnehmerstaaten - wirtschaftliche und ökologische Fragen verstärkt in den Fokus rückte. Kasachstan machte sich diese Dynamik zunutze, um den eigenen Ambitionen zu diesen Themen mehr Gehör zu verschaffen. Infolgedessen verstärkten sich die Bestrebungen und Erwartungen des Landes bezüglich der Einbeziehung des Themas Konnektivität in die OSZEAgenda. Zwischen 2016 und 2019 rückten Deutschland, Österreich, Italien und die Slowakei unter ihrem Vorsitz jeweils unterschiedliche Schwerpunkte des Themenfeldes in den Mittelpunkt. Italien stellte zum Beispiel die Digitalisierung in den Vordergrund, während der deutsche Vorsitz Konnektivität eng mit guter Regierungsführung verknüpfte. ${ }^{18}$

Kasachstans Ambitionen im Bereich der Konnektivität gipfelten in seiner Lobbyarbeit zur Einrichtung eines OSZEThemenzentrums für nachhaltige Konnektivität (Centre on Sustainable Connectivity, CSC) in der Hauptstadt Nur-Sultan. Diese Idee wurde im Juni 2017 beim zweiten Vorbereitungstreffen zum Wirtschafts- und Umweltforum der OSZE in der Hauptstadt erstmalig vorgestellt und im Dezember auf dem Wiener Treffen des Ministerrats weiter diskutiert. Zwölf Monate später, anlässlich des Ministerrats 2018 in Mailand, brachte der kasachische Außenminister Kairat Abdrachmanow erneut den Vorschlag zur Errichtung eines 
CSC ein. Im Juli 2019 wurde der Vorschlag beim informellen OSZE-Ministertreffen in der Slowakei weiter konkretisiert, als der neue Außenminister Beibut Atamkulow erklärte, das Zentrum solle zur „Konnektivität im weiteren Sinne“ forschen. Kairat Sarybay, der kasachische Botschafter bei der OSZE, konkretisierte das mögliche Aufgabenspektrum des CSC durch Themen mit Bezug zur zweiten Dimension wie „Fragen der nachhaltigen Konnektivität, [...] gute Regierungsführung, Green Economy, Entwicklung neuer Technologien, Katastrophenschutz, Energiesicherheit und Handelsförderung “. ${ }^{19}$ Die kasachische Regierung schlug vor, diese Institution im Astana International Financial Centre (AIFC) einzurichten, einem Finanzzentrum in Nur-Sultan auf halbem Weg zwischen Flughafen und Stadtzentrum, das Mitte 2018 offiziell eröffnet worden war. ${ }^{20}$ Der Standort soll Synergien zwischen dem CSC und internen Institutionen wie auch Wissenschaftlern der nahe gelegenen Nasarbajew-Universität ermöglichen. Die konkreten Aufgaben eines solchen CSC wurden allerdings noch nicht festgelegt.

Der ursprüngliche Vorschlag Kasachstans, die Kosten für den Betrieb des CSC selbst zu übernehmen und/oder eine Gruppe von „Freunden Kasachstans“ dafür aufkommen zu lassen, wird seit einiger Zeit nicht mehr vorgebracht. Neuerdings besteht Kasachstan darauf, dass die Kosten aus dem Gesamthaushalt der OSZE getragen werden. ${ }^{21}$ Es gibt Grund zu der Annahme, dass dieser Sinneswandel nicht in erster Linie auf finanziellen Überlegungen beruht. Angesichts des knappen Budgets der OSZE, welches aufgrund des nominellen Nullwachstums effektiv schrumpft, ist es unwahrscheinlich, dass sowohl das derzeitige Programmbüro als auch ein CSC gleichzeitig betrieben werden können, wenn die Mittel dafür aus dem Gesamthaushalt kommen sollen. Während die südosteuropäischen Länder einen großen Anteil des OSZE-Gesamthaushalts (34\%) erhalten, ist der Anteil Zentralasiens ebenfalls nicht unerheblich (15\%) und übertrifft sowohl Osteuropa (4\%) als auch den Südkaukasus (2\%). ${ }^{22}$ Aus diesem Grund und weil momentan keine Einsätze zur Friedensstiftung, zur Friedenserhaltung sowie zur unmittelbaren Konfliktprävention benötigt werden, ist eine wesentliche Erhöhung des auf Zentralasien entfallenden Anteils auf absehbare Zeit unwahrscheinlich. Der ehemalige OSZE-Generalsekretär Thomas Greminger hatte bereits darauf hingewiesen, dass die OSZE angesichts des knappen Gesamthaushalts nicht in der Lage sein werde, zwei Büros in Kasachstan zu unterhalten. Der kasachische Vorschlag, das CSC aus dem Gesamthaushalt zu bezahlen, bedeutet insofern unweigerlich, dass das Programmbüro in Nur-Sultan geschlossen werden müsste, wenngleich dies so nicht öffentlich erklärt wurde.

\section{Normumgehung und Führungsambitionen}

Abgesehen von der Aussicht auf unmittelbare materielle Vorteile ist der Vorschlag, ein Konnektivitätszentrum im Land einzurichten, für Kasachstan auch ein Mittel, um ungewollten normativen 
Verpflichtungen zu entgehen und seinen Führungsambitionen vor Ort mehr Geltung zu verschaffen. Wie oben angemerkt, könnte es sein, dass beabsichtigt ist, das derzeitige OSZE-Programmbüro in Nur-Sultan durch das CSC zu ersetzen. Bereits bei der Plenarsitzung des Ministerrats 2014 in Basel deutete der stellvertretende kasachische Außenminister Alexej Wolkow an, dass das Büro gänzlich geschlossen werden könnte. Diese Drohung wurde zwar nicht umgesetzt, ${ }^{23}$ jedoch brachte die kasachische Regierung später wiederholt die Änderung des Mandats der Mission ins Gespräch. Die zweite Dimension wurde dadurch deutlich herausgehoben, während die beiden anderen Dimensionen mindestens heruntergespielt, wenn nicht gar völlig ignoriert wurden. Wie bei den meisten anderen Präsenzen in Zentralasien wurde auch die erste, 1998 eingerichtete OSZE-Feldoperation in Kasachstan inzwischen auf das derzeitige Programmbüro zurückgefahren. Zudem büßte das Büro im Jahr 2015 weitere Zuständigkeiten ein, zum Beispiel die Prozessbeobachtung, also die Beobachtung von Gerichtsverhandlungen und -verfahren und das Sammeln von Informationen, um beurteilen zu können, inwieweit diese den Standards für faire Verfahren entsprechen. ${ }^{24}$ Bereits 2004 gehörte Kasachstan zu den prominenteren Unterzeichnern des „Astana-Appells“, in dem die angeblich einseitige Betonung der menschlichen Dimension durch die OSZE und die damit einhergehende Einmischung in innere Angelegenheiten beklagt wurde. Im Jahr 2018 erklärte AuBenminister Kairat Abdrachmanow als erster Vertreter der kasachischen Regie- rung schriftlich, das Programmbüro habe sein Mandat erfüllt und ein neuer Schwerpunkt sei nun gefordert. ${ }^{25}$

Offenbar sieht die kasachische Regierung die Aufnahme einer offiziellen OSZE-Institution wie des vorgeschlagenen CSC als eine Möglichkeit, ihr OSZEEngagement neu auszurichten. Möglicherweise betrachtet sie die CSC-Initiative auch als willkommene Ablenkung von unangenehmen Verpflichtungen, insbesondere aus dem Bereich der dritten (menschlichen) Dimension. ${ }^{26}$ Diese Denkweise spiegelt zugleich eine allgemeinere Tendenz innerhalb der OSZE wider: Mehrere Teilnehmerstaaten sträuben sich zunehmend gegen die Einhaltung von OSZE-Verpflichtungen und blockieren mit ihrem Vetorecht die Einstellung unliebsamer hochrangiger OSZE-Vertreter. ${ }^{27}$

Kasachstans Bemühungen um Konnektivität sind auch durch seine Führungsambitionen motiviert. Seit den 1990er Jahren hat das Land in zahlreichen Kooperationsinitiativen große regionale und internationale Führungsstärke bewiesen. So schlug der erste Präsident Kasachstans, Nursultan Nasarbajew, unter anderem vor, dem Modell der europäischen Integration zu folgen und eine Integration im Rahmen einer Eurasischen Union anzustreben. ${ }^{28}$ Daneben ist auch der kasachische OSZE-Vorsitz im Jahr 2010 ein Zeichen von Führungsstärke. ${ }^{29}$ Das Land ebnete in dieser Zeit maßgeblich den Weg für die Erklärung einer atomwaffenfreien Zone in Zentralasien. Insbesondere hat sich Kasachstan mit einigem Erfolg und einer ehrgeizigen diplomatischen und außen- 
politischen Agenda vor allem als Friedensvermittler positioniert, zum Beispiel als Gastgeber des Prozesses zur Beilegung des Syrien-Konflikts. ${ }^{30}$ Es ist vielleicht kein Zufall, dass Kasachstan auf dem bereits erwähnten informellen OSZE-Ministertreffen in der Slowakei 2019 nicht nur den Vorschlag für die Einrichtung eines CSC publik machte, sondern sich auch als Ort für ein Treffen zur Beilegung des Afghanistankonflikts ins Gespräch brachte. ${ }^{31}$ Das Land ist somit in beschränktem Maße zu einem Garanten für regionale und internationale Sicherheit avanciert. Die Konnektivitätsambitionen in Nur-Sultan und Wien zielen außerdem darauf $\mathrm{ab}$, Orientierung zu geben und die zunehmend proaktive Rolle Kasachstans in der regionalen und internationalen Politik zu festigen. Mit der Gründung des International Financial Centre, in dem das CSC nach der Vorstellung Kasachstans untergebracht wäre, verknüpfte die kasachische Regierung die Hoffnung, das Land zu einer Drehscheibe des globalen Finanzsystems zu machen. ${ }^{32}$ Nach dem Vorbild früherer Initiativen, insbesondere am Persischen Golf, beschreibt es sich selbst als „globales Geschäfts- und Finanzzentrum, das die Volkswirtschaften [...] Zentralasiens, des Kaukasus, der EAWU, Westchinas, der Mongolei, des Nahen Ostens und Europas miteinander verbindet. “"33

Jedoch ist die Führungsrolle Kasachstans nicht unangefochten. Usbekistan und Kirgisistan haben sich ebenfalls als Gastgeber von Afghanistan-Gesprächen angeboten, um eine Führungsrolle zu übernehmen. Usbekistan, das bevölkerungsreichste der fünf zentralasiatischen
Länder, ist generell skeptisch gegenüber den Führungsambitionen Kasachstans. Die Einrichtung eines CSC würde diese Wahrnehmung noch verstärken. ${ }^{34}$ In den weiteren Gesprächen über diese Initiative wäre Kasachstan daher gut beraten, die Vorbehalte seiner südlichen Nachbarn ernst zu nehmen und zu versuchen, sie auszuräumen.

Kasachstans Konnektivitätsagenda ist auch durch Statusdenken motiviert, verbunden mit normativem Eskapismus und dem Streben nach internationalem Ansehen. Zunehmend verstärkt sich der Eindruck, als ob das OSZE-Programmbüro von Kasachstan als Beleg für eine (nach kasachischer Lesart falsche) Wahrnehmung gesehen wird, nach der die innenpolitische Situation unzulänglich sei. Dieses Unbehagen ist insoweit verständlich, als sich Kasachstan in mehrfacher Hinsicht positiv von einigen anderen Ländern in der Region (wie Turkmenistan und Tadschikistan) abhebt, etwa in Bezug auf das Investitionsklima, den wirtschaftlichen Fortschritt, die Rechtsstaatlichkeit und die politische Stabilität. Vor diesem Hintergrund entspricht das vorgesehene CSC dem Wunsch Kasachstans, eine normale OSZE-Institution statt eines Büros für Teilnehmerstaaten mit "echtem Bedarf“ - im Land zu haben. Auf dem informellen OSZE-Treffen 2019 in der Slowakei erklärte die kasachische Delegation entsprechend, die CSC-Initiative ziele auch auf eine ausgeglichenere geographische Verteilung von OSZE-Institutionen ab. ${ }^{35}$

$\mathrm{Ob}$ das CSC zustande kommen wird, ist schwierig vorherzusagen. Eine ausreichende Finanzierung vorausgesetzt, 
müsste der Ständige Rat im Konsens aller 57 Teilnehmerstaaten darüber entscheiden. Maßgebliche Unterstützung aus den übrigen vier zentralasiatischen Staaten fehlt bislang. ${ }^{36}$ Darüber hinaus zögern etliche andere Teilnehmerstaaten bisher noch, die Idee mitzutragen, darunter mindestens ein größerer EU-Staat. Für Kasachstan und andere Interessierte ist es daher wichtig zu klären, welche Aufgaben das CSC übernehmen soll und zu versuchen, denjenigen Staaten entgegenzukommen, die bislang noch zögerlich in ihrer Unterstützung sind.

\section{Schlussfolgerungen und Empfehlungen}

Der OSZE kommt in Sachen Konnektivität eine wichtige Rolle zu, und Kasachstan hat bei der regionalen und internationalen Zusammenarbeit und Stabilität bemerkenswerte Führungsstärke bewiesen. Seine Konnektivitätsinitiative ist daher ernst zu nehmen. Die Unterstützung der kasachischen Ambitionen darf jedoch nicht zu einer Vernachlässigung der Verpflichtungen aus der ersten und dritten Dimension führen. Deshalb sollte das Spillover-Potenzial in allen drei Dimensionen genutzt werden. Zwar gehört Konnektivität streng genommen in den Themenbereich der zweiten Dimension. Soweit sie politisches Vertrauen und Transparenz schafft und ein besseres Grenzmanagement fördert, betrifft sie jedoch auch die erste Dimension. Darüber hinaus hat sie Auswirkungen auf die dritte, die menschliche Dimension der Sicherheit. Tatsächlich verweisen sowohl die Bonner Erklärung von
1990 als auch der Beschluss des Ministerrats von 2016 direkt auf die Achtung der Menschenrechte. Auch das Thema gute Regierungsführung, das Auswirkungen auf die dritte Dimension hat, findet häufiger Beachtung, seit der deutsche Vorsitz es explizit in den Vordergrund der OSZE-Diskussionen zur Konnektivität gerückt hat. Obwohl die OSZE das Thema der guten Regierungsführung vorwiegend durch die wirtschaftliche Brille (Bekämpfung von Korruption und Geldwäsche) betrachtet, fordert sie in diesem Zusammenhang auch die volle Einhaltung von Rechtsstaatlichkeit. ${ }^{37}$ Implizit sind damit Ausstrahlungseffekte auf die dritte Dimension verbunden, weil die OSZE Rechtsstaatlichkeit als Grundpfeiler der Menschenrechte und der Demokratisierung betrachtet. Politisch-militärische und menschliche Sicherheitsfragen haben weiterhin zentrale Bedeutung für den ganzheitlichen und nachhaltigen Konnektivitätsbegriff der OSZE. Dies sollte sich auch nicht ändern.

Die OSZE mit ihren begrenzten Ressourcen ist nicht die bedeutendste Organisation im Bereich der Konnektivität, vor allem im Vergleich mit Institutionen wie der EU, der Weltbank, der Welthandelsorganisation, der Europäischen Bank für Wiederaufbau und Entwicklung und der UN-Wirtschaftskommission für Europa, die allesamt mehr Erfahrung und Ressourcen haben. Entsprechend ihrem begrenzten Einfluss auf diesem Gebiet sollte die OSZE daher als Plattform, Diskussionsforum oder Wissensvermittler für Konnektivitätsprojekte im OSZE-Raum fungieren. ${ }^{38}$ Über diese Funktionen kann die OSZE einen Mehrwert beisteuern. 
Das vorgeschlagene CSC könnte vor allem als Denkfabrik oder Clearingstelle für nachhaltige Handels- und Verkehrsprojekte ausgestaltet werden und dazu beitragen, Defizite in den Bereichen ökologische Nachhaltigkeit, Wirtschaftlichkeit, Handelsstandards sowie Transparenz und Verantwortung zu vermeiden oder abzumildern. Die Anfälligkeit für Überschuldung ist ein weiteres wichtiges Thema - nicht so sehr in Kasachstan, sondern vielmehr in anderen Teilnehmerstaaten wie Kirgisistan, Tadschikistan und der Mongolei. ${ }^{39}$ Ein Konnektivitätszentrum könnte hier beispielsweise anhand einschlägiger Studien potenzielle Kreditnehmer für künftige Projekte beraten, Schuldenprobleme angehen und Standards für nachhaltige Finanzierung festlegen helfen. Darüber hinaus könnte das CSC mit Studien, Workshops und Konferenzen den Handel und Verkehr im OSZE-Raum ankurbeln. Dies wäre namentlich für die fünf zentralasiatischen Länder von Bedeutung, die ihre Handelsund Wirtschaftskooperation seit 2014 dank mehrerer Infrastrukturprojekte (wie der Gaspipeline von Turkmenistan über Usbekistan und Kasachstan nach Westchina, die zwischen 2006 und 2009 gebaut wurde) deutlich ausgeweitet haben und weitere Projekte planen. ${ }^{40} \mathrm{Da}$ die OSZE bei einigen Aspekten der Konnektivität nur bescheidene Finanzmittel und begrenztes Fachwissen hat, müsste ein CSC eng mit anderen Entwicklungs-, Infrastruktur- und Finanzinstitutionen zusammenarbeiten.

Wenn sich die OSZE im Bereich Konnektivität profilieren will, sollte sie auch ihren Mehrwert als Sicherheitsorganisati- on - im Vergleich zu eher technisch geprägten Organisationen - zunutze machen und den Zusammenhang zwischen Konnektivität und Sicherheit hervorheben. Grenzmanagement, vertrauensbildende Maßnahmen, Vereinbarungen zur Grenzziehung und zur Lösung grenzüberschreitender Probleme, aber auch die Energiesicherheit, wären wichtige Themen für ein künftiges CSC.

Bei seinen Bemühungen für die Einrichtung eines Konnektivitätszentrums im eigenen Land sollte Kasachstan vermeiden, andere zentralasiatische Länder mit seinen Führungsambitionen in diesem Bereich zu verprellen. Seit 2016 hat sich Usbekistan als „doppelter Binnenstaat" ${ }^{\text {"41 }}$ ebenfalls $\mathrm{zu}$ einem engagierten Befürworter der Konnektivität entwickelt und hierbei die Beziehungen zwischen den zentralasiatischen Ländern besonders hervorgehoben. ${ }^{42}$ Kasachstan sollte bei jeder Initiative zur Förderung der Konnektivität andere interessierte zentralasiatische Staaten einbinden. Dies ließe sich unter anderem durch die zahlenmäßige Berücksichtigung von Experten aus Usbekistan in ein mögliches CSC erreichen. Insgesamt sollten Kasachstan und andere Interessierte die Funktionen des CSC detaillierter erörtern und versuchen, denjenigen Staaten entgegenzukommen, die einem solchen Projekt zögerlich gegenüberstehen.

\section{Notizen}

1 OSCE, „Connectivity“, https://www.osce. org/oceea/446224. 
Die Analyse basiert auf Primär- und Sekundärquellen sowie Hintergrundinterviews aus den Monaten Juli und August 2020.

3 Vgl. Stefan Wolff, Economic diplomacy and connectivity: What role for the OSCE? University of Birmingham, Institute for Conflict, Cooperation and Security, 2018, S. 6, https://www.birmingham .ac.uk/Documents/college-social-sciences/ government-society/iccs/news-events/201 8/Osce-Report.pdf.

4 Robert O. Keohane/Joseph Nye, Power and Interdependence: World Politics in Transition, Boston: Little, Brown, 1977.

Z.B. Nino Kemoklidze/Stefan Wolff, „Trade as a Confidence-Building Measure in Protracted Conflicts: The Cases of Georgia and Moldova Compared", in: Eurasian Geography and Economics 3/2020, S. 305-332; Erik Gartzke/Quan $\mathrm{Li} /$ Charles Boehmer, Investing in the Peace: Economic Interdependence and International Conflict, in: International Organization 2/2001, 391-438.

Keohane/Nye, oben zitiert (Anm.4), S. 14-16.

Nadine Godehardt/Karoline PostelVinay, „Connectivity and Geopolitics: Beware the ,new wine in old bottles' approach“, SWP Comment 2020/C 35, 2020, S. 4-5, https://www.swp-berlin.org/ en/publication/connectivity-and-geopolit ics-beware-the-new-wine-in-old-bottles-ap proach/.

OSZE, Dokument der Bonner Konferenz über wirtschaftliche Zusammenarbeit in Europa, 1990, S. 2-4, https://www.osce.or g/de/eea/14083

OSZE, Abschlussdokument des Elften Treffens des OSZE-Ministerrats, MC.DOC/1/03, 2. Dezember 2003, S. 17, https://www.osce.org/mc/40533.

OSCE, „OSCE economic and environmental activities“, https://www.osce.org loceea.
11 OSCE/UNECE, Handbook of Best Practices at Border Crossings - A Trade and Transport Facilitation Perspective, 2012, https://www.osce.org/files/f/docu ments/7/6/88238.pdf.

12 OSCE/UNECE, Inland Transport Security Discussion Forum Proceedings, 2012, https://www.osce.org/files/f/documents/b /6/99852.pdf.

13 Richard Pomfret, The Central Asian Economies in the Twenty-First Century: Paving a New Silk Road, Princeton and Oxford: Princeton University Press, 2019, S. 215-223.

14 Evgeny Vinokurov, Introduction to the Eurasian Economic Union, Cham: Palgrave Macmillan, 2018, S. 134-137.

15 Nargis Kassenova, „China's Silk Road and Kazakhstan's Bright Path: Linking Dreams of Prosperity“, in: Asia Policy 24/2017, S. 111-112.

16 Sébastien Peyrouse/Gaël Raballand, „Central Asia: The New Silk Road Initiative's Questionable Economic Rationality“, in: Eurasian Geography and Economics 4/2015, 405-420.

17 Pál Dunay, „Kasachstans einzigartiger OSZE-Vorsitz 2010“, in: IFSH (Hrsg.), OSZE-Jahrbuch 2011, S. 60, https://ifsh.d e/file-CORE/documents/jahrbuch/11/Du nay-dt.pdf.

18 Franziska Smolnik, „Cooperation, Trust, Security? The Potential and Limits of the OSCE's Economic and Environmental Dimension“, SWP Research Paper 2019/RP 16, 2019, S. 17-19, https://www. swp-berlin.org/en/publication/the-potent ial-and-limits-of-the-osces-economic-andenvironmental-dimension/.

19 Elya Altynsarina, „Kazakhstan announces initiatives to reinforce OSCE goals", The Astana Times, 11 July 2019, https://astana times.com/2019/07/kazakhstan-announce s-initiatives-to-reinforce-osce-goals/.

20 Unternehmen und Institutionen, die beim AIFC registriert sind (derzeit fast 
600), profitieren von Steuervergünstigungen, einer modernen Finanzregulierung und einem Handelsrecht, das auf dem englischen Recht basiert. Das AIFC hat zudem Schlichtungs- und Streitbeilegungsmechanismen eingeführt, die als Modell für den Rest des Landes dienen können. So will die Regierung das Investitionsklima verbessern und Investoren nach Kasachstan locken. Siehe Nicolás Zambrana-Tévar, „The new Court of the Astana International Financial Centre: Promoting the Rule of Law or Giving up on the Rule of Law?", in: Central Asia Business Journal, Autumn/2019, 37-48.

21 Interview mit einem OSZE-Mitarbeiter aus Zentralasien, Juli 2020.

22 Die Zahlen basieren auf dem Gesamthaushaltsplan 2019. Siehe OSCE, Annual Report 2019, S. 98, https://www.osce.org/ annual-report/2019.

23 Die Regierung hätte dies grundsätzlich tun können, da das Mandat für jede Feldmission einmal im Jahr durch den Ständigen Rat einvernehmlich verabschiedet werden muss. So bewirkte die aserbaidschanische Regierung beispielsweise im Jahr 2016 mithilfe ihres Vetorechts die Schließung des OSZE-Büros in Baku, das ihr ein Dorn im Auge war.

24 Sebastian Mayer, „Walking Alone, Walking Together? OSCE-EU relations in Central Asia“, Policy Brief \#62, OSCE Academy Bishkek, 2020, S. 3-4, http://osc e-academy.net/upload/file/62.pdf.

25 Interview mit einem OSZE-Mitarbeiter aus Zentralasien, Juli 2020.

26 Das derzeitige OSZE-Programmbüro in Nur-Sultan deckt alle drei Dimensionen ab.

27 Walter Kemp, „Executed structures: Leadership crisis in the OSCE“, in: Security and Human Rights Monitor, 14 July 2020, https://www.shrmonitor.org/executed-structures-leadership-crisis-in-the-osce/.
28 Assylzat Karabayeva, „Leaders, Ideas, and Norm Diffusion in Central Asia and Beyond“, in: Asian Journal of Comparative Politics 2019, Volume 6, Issue 1, March 2021, 25-44, https://doi.org/ $10.1177 / 2057891119887813$.

29 Dunay, oben zitiert (Anm. 17), S. 57.

30 Rachel Vanderhill/Sandra F. Joireman/ Roza Tulepbayeva, „Between the Bear and the Dragon: Multivectorism in Kazakhstan as a Model Strategy for Secondary Powers", in: International Affairs 4/2020, S. 986-990.

31 Altynsarina, oben zitiert (Anm. 19).

32 Sir Suma Chakrabarti, „EBRD’s vision for Astana International Financial Centre“, The Astana Times, 25 May 2016, https://astanatimes.com/2016/05/ebrds -vision-for-astana-international-financial-c entre/.

33 Astana International Financial Centre, https://aifc.kz/.

34 Interview mit einem OSZE-Mitarbeiter aus Zentralasien, Juli 2020.

35 Altynsarina, oben zitiert (Anm. 19).

36 Interview mit einem OSZE-Beamten aus Zentralasien, Juli 2020.

37 OSCE, „Good governance“, https://www. osce.org/oceea/446335.

38 Vgl. Wolff, oben zitiert (Anm. 3), S. 2427.

39 John Hurley/Scott Morris/Gailyn Portelance, „Examining the debt implications of the Belt and Road Initiative from a policy perspective“, CGD Policy Paper 121, Center for Global Development, March 2018, S. 16-19, https://www.cgdev .org/sites/default/files/examining-debt-im plications-belt-and-road-initiative-policyperspective.pdf.

40 Richard Pomfret, „Kooperation im Politikfeld Handel“, in: Jakob Lempp/Sebastian Mayer/Alexander Brand (Hrsg.), Die politischen Systeme Zentralasiens: Interner Wandel, externe Akteure, regionale 
Kooperation, Wiesbaden: Springer VS, 2020, 318-322.

41 D.h., es ist ausschließlich von Ländern umgeben, die selbst keinen Zugang zum Meer haben.

42 Fabio Indeo, „New Trends in Central Asian Connectivity“, in: Carlo Frappi/ Fabio Indeo (Hrsg.), Monitoring Central Asia and the Caspian Area: Development
Policies, Regional Trends, and Italian Interests, Eurasiatica 13, Venice: Edizioni Ca'Foscari, 2019, S. 65-80; Richard Weitz, „Uzbekistan's new foreign policy", Central Asia-Caucasus Institute and Silk Road Studies Program, 2018, S.912, 31-42, https://silkroadstudies.org/reso urces/pdf/SilkRoadPapers/1801Weitz.p df. 